FORMATION Formation emploi

Revue française de sciences sociales

102 | avril-juin 2008

Pêle-mêle

\title{
Une nouvelle dynamique des marchés du travail réglementés, à l'aune de trois professions
}

Recent dynamics on regulated labour markets viewed in the light of three occupations

Nueva dinámica de los mercados de trabajo reglamentados, según tres profesiones

Neue Dynamik reglementierter Arbeitsmärkte an drei Berufen veranschaulicht

\section{Anne Moysan-Louazel et Gérard Podevin}

\section{(2) OpenEdition}

Journals

Édition électronique

URL : http://journals.openedition.org/formationemploi/2181

DOI : 10.4000/formationemploi.2181

ISSN : 2107-0946

Éditeur

La Documentation française

Édition imprimée

Date de publication : 1 juin 2008

Pagination : 5-19

ISSN : 0759-6340

Référence électronique

Anne Moysan-Louazel et Gérard Podevin, «Une nouvelle dynamique des marchés du travail réglementés, à l'aune de trois professions », Formation emploi [En ligne], 102 | avril-juin 2008, mis en ligne le 01 juin 2010, consulté le 30 octobre 2020. URL : http://journals.openedition.org/ formationemploi/2181 ; DOI : https://doi.org/10.4000/formationemploi.2181 


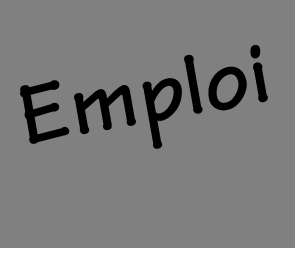

\title{
Une nouvelle dynamique des marchés du travail réglementés, à l'aune de trois professions'
}

Par Anne Moysan-Louazel et Gérard Podevin*

\begin{abstract}
Reconsidérer la dynamique des marchés réglementés implique de s'ouvrir à de nouvelles pratiques, à de nouvelles carrières, à de nouvelles possibilités de mobilité et pas seulement d'élargir les voies d'accès.
\end{abstract}

Sur la période récente, de profondes transformations touchent le fonctionnement des marchés du travail fermés propres à certaines professions. Elles se traduisent notamment par l'adoption de nouvelles règles permettant de répondre aux tensions rencontrées sur ces marchés tout en assurant le maintien de leur « fermeture $»^{2}$. Par « fermeture » ou « clôture »,

${ }^{1}$ L'article s'appuie d'une part sur divers travaux portant sur le marché du travail à la pêche menés par le centre associé au Céreq de Rennes depuis cinq ans; d'autre part, il exploite une volumineuse documentation sur les professions de la santé, en particulier sur la profession d'infirmière, tant à partir d'études sociologiques portant sur les changements dans l'activité et la professionnalisation de cette profession, que de données démographiques issues de travaux de l'ONDPS (Observatoire national de la démographie des professions de santé) ou de la DRESS (Direction de la Recherche, des Études, de l'Évaluation et de la Statistique du ministère de la Santé) Une partie de ces données a fait l'objet d'une communication lors des journées de l'AES de 2005 (A. Moysan-Louazel, G. Podevin, 2005). Enfin, concernant les experts-comptables, l'article s'appuie sur des études et rapports issus du Conseil supérieur de l'Ordre des experts-comptables ainsi que de diverses organisations professionnelles. Il exploite aussi la presse professionnelle de ce secteur.

${ }^{2}$ Ces tensions ne sont pas propres aux marchés professionnels fermées, mais cette fermeture, particulièrement dans le cas de réglementations qui en contrôlent strictement l'accès, rend ici la recherche de nouvelles régulations beaucoup plus problématique. nous entendons les restrictions et le contrôle dans l'accès à certaines professions, la garantie de la compétence technique et l'encadrement des modes d'exercice de l'activité principalement par l'action réglementaire de l'État (Demazière, 2008). Toutefois, la mise en place de nouvelles règles se réalise aujourd'hui suivant des modalités variées, conjuguant augmentation des flux d'entrée, ouverture à de

* Anne Moysan-Louazel est économiste, maître de conférences, chercheur au Crem (Centre de recherche en économie et management) à l'université de Rennes 1 . Ses principaux axes de recherche portent sur la carrière des cadres (particulièrement des ingénieurs et des chercheurs) et sur le marché de la formation continue. Elle est co-auteur d'un rapport sur "L'intermédiation dans les relations d'emploi au travers des exemples du portage salarial et de l'intérim hautement qualifié : regards croisés en économie du travail et en droit social », Dares (2005). Elle coordonne actuellement une étude nationale sur l'activité formation continue dans les IUT IInstitut universitaire de technologie). 
Gérard Podevin est économiste et démographe, ingénieur de recherche au Céreq et Chargé de mission au centre associé de Bretagne. II est co-auteur d'une récente étude sur "L'emploi et la formation dans le secteur du tourisme " et, en collaboration avec Anne Moysan-Louazel et al., d'un rapport sur "Le territoire, lieu d'élaboration et d'expérimentation de nouvelles normes collectives: l'exemple des Fongécif», qui donnera lieu prochainement à une publication aux PUR. II a été le coordinateur du premier Contrat d'étude prospective (CEP) sur le secteur des pêches maritimes, publié à la Documentation française en 2003. II réalise actuellement une évaluation d'impact de ce CEP.

nouveaux publics, réforme des systèmes de formation, formes renouvelées de mobilité en cours de carrière, ou bien encore changements importants dans la professionnalisation des métiers correspondants. Ces changements sont visibles pour les professions réglementées auxquelles l'article s'intéresse plus particulièrement. À l'heure de la libre prestation de services, de la libre concurrence et de la libre circulation des travailleurs dans l'espace communautaire, la réglementation des services offerts et le mode d'accès à ces marchés du travail suscitent immanquablement de nombreux débats et analyses.

Pour illustrer ces changements, nous proposons d'étudier trois professions réglementées, en apparence très éloignées, mais qui ont en commun d'être soumises à des tensions les obligeant à revoir, suivant des logiques voisines, leur mode de régulation, d'ajustement et de professionnalisation. Il s'agit des professions de marins-pêcheurs, d'infirmières et d'experts-comptables. Leur point commun réside dans les règles qui en contrôlent l'accès, celui-ci n'étant possible qu'aux titulaires d'une certification bien définie et obligatoire. Pour les entreprises, des tensions se cristallisent principalement sous forme de difficultés à recruter et à stabiliser leur main-d'œuvre ; cependant, ces difficultés ne traduisent pas seulement des déséquilibres entre offres et demandes mais révèlent aussi les limites actuelles de formes institutionnelles spécifiques qui peinent à s'optimiser dans un environnement économique et démographique renouvelé. Aussi, ces tensions ne doivent-elles pas être considérées du seul point de vue quantitatif d'une conjoncture démographique défavorable, qu'une meilleure gestion anticipée des flux viendrait régler. Elles supposent également de revoir les régulations qui sous-tendent le fonctionnement de ces marchés, et qui ne paraissent plus adaptées aux enjeux actuels de renouvellement démographique et sociologique de leurs actifs, de professionnalisation et d'harmonisation européenne.

Pour autant, comme nous tenterons de le montrer, les changements qui affectent certaines règles ne remettent pas en cause le caractère fermé de ces marchés. Les barrières à l'entrée, qui contrôlent l'accès à ces professions, demeurent, voire se renforcent. C'est pourquoi, si nous parlons d' ' ouverture » c'est pour souligner certaines ruptures dans les pratiques de gestion des recrutements ou des mobilités, et la volonté des acteurs de rechercher, autour de règles obligées, de nouvelles marges de manœuvre.

Dans une première partie, nous présentons l'arrièreplan théorique qui nous permet de rendre compte des marchés fermés et de leur dynamique. Dans une seconde partie, nous examinons l'évolution récente des trois marchés étudiés en montrant pourquoi et comment ils sont contraints à repenser leurs réglementations sans toutefois pouvoir renoncer à leur fermeture. Un des constats tiré d'une lecture transversale des évolutions constatées sur chacun des marchés étudiés, sous l'effet de jeux d'acteurs en recomposition, montrera que la thèse défendue par C. Paradeise (1988), selon laquelle le travail de « clôture » des marchés est en perpétuel recommencement et les monopoles du marché du travail toujours précaires, est encore d'actualité.

\section{LES PROFESSIONS RÉGLEMENTÉES : DES MARCHÉS DU TRAVAIL « FERMÉS » SPÉCIFIQUES}

Le concept de «marché du travail fermé » a fait l'objet de nombreux travaux d'économistes et de sociologues qui ont permis d'identifier les caractéristiques-clés de ces segments de marché. Dès les années 70, la théorie du marché du travail segmenté, approche théorique non traditionnelle du marché du 


\section{Encadré 1 \\ Méthodologie}

Les informations sur lesquelles repose l'étude comparative des trois professions sont issues pour une part de travaux d'étude réalisés par les auteurs (plus particulièrement sur le secteur des pêches maritimes et celui des professions médicales et médico-sociales), complétés et réactualisés par une série d'entretiens auprès de responsables de l'administration en charge du pilotage de la formation de ces professions, auprès d'employeurs, et auprès d'acteurs ayant une expertise reconnue sur ces champs professionnels. Pour le secteur des pêches maritimes, outre des travaux conduits par le Céreq dans le cadre du Contrat d'étude prospective rendu en 2002, une dizaine d'entretiens ont été réalisés en 2006 et 2007 auprès de la Direction des Affaires maritimes et des Gens de mer, de responsables de formation, d'armateurs, d'organismes financeurs de la formation, et de comités locaux des pêches.

Pour les infirmières, les informations proviennent de nombreux rapports publiés par la DRESS (Direction de la Recherche, des Études, de l'Évaluation et de la Statistique du ministère de la Santél, I'ONDPS (Observatoire national de la démographie des professions de santé). Ces matériaux ont également été complétés par des enquêtes de terrain, visant à actualiser, valider, et préciser certaines données, auprès de responsables de formation et de chefs d'établissements.

Concernant les experts-comptables, les documents ont été fournis par le Conseil supérieur de l'Ordre des experts-comptables. Les enquêtes ont été réalisées auprès d'experts-comptables, de représentants de l'Ordre et de responsables d'associations professionnelles.

L'organisation de l'ensemble des informations collectées s'est structurée principalement autour de trois axes: évolution des conditions d'entrée dans la profession, évolution du système de formation, construction des trajectoires professionnelles en cours de carrière et changement dans les pratiques de professionnalisation.

travail initiée par Doeringer et Piore (1971), s'est structurée autour des concepts de marché interne et de marché externe. Selon ces auteurs, le marché du travail se ferme sur ce qu'ils appellent des marchés internes du travail, internes à l'entreprise et/ou internes à une profession ${ }^{3}$. En première lecture, l'intérêt du concept de marché fermé est de prendre en compte, dans une vision globale, à la fois les «faits organisationnels» et les «faits de socialisation » (Lamanthe, 2005) à la place des mécanismes standards d'offre et de demande et de la détermination simultanée des prix et des quantités. Cependant, à cette vision fortement bi-polaire peut être opposée une approche de type « continuum » qui postule que

\footnotetext{
${ }^{3}$ Rappelons que Doeringer et Piore définissent le marché interne du travail comme « une unité administrative, où la rémunération et l'allocation du travail sont gouvernées par un ensemble de règles et procédures administratives ; il doit être distingué du "marché externe du travail" de la théorie économique conventionnelle où les décisions de rémunération, d'allocation et de formation sont contrôlées directement par des variables économiques ».
}

tous les marchés du travail sont organisés mais qu'ils se distinguent par leur mode d'organisation. Le Corre (2001) a ainsi souligné que «contrairement à la catégorie du marché externe, conceptualisée par les économistes du travail, ce n'est pas l'absence de règles (i.e le marché) qui signale l'existence d'un marché du travail ouvert, mais bien l'absence de règles protectrices $»$.

C'est aussi la thèse défendue par C. Paradeise, depuis vingt ans, dans l'ensemble de ses travaux. Selon cet auteur, tous les marchés du travail sont des combinaisons d'organisation et de marché qu'elle différencie selon leur mode de régulation (la loi, le règlement, la convention et la coutume) et le niveau d'organisation (le secteur, le métier, la branche et l'entreprise). La fermeture d'un marché du travail renvoie à l'idée de « sa monopolisation par un collectif de travailleurs auxquels est socialement reconnue la possession de savoirs et savoir-faire jugés indispensables à l'élaboration d'un bien ou d'un service » (Paradeise, 1988). 
La définition, la construction et l'entretien de la qualification d'une main-d'œuvre pour une tâche déterminée sont bien le trait commun à tous les « marchés fermés », qui fonde et justifie leur clôture. Celle-ci va se manifester par la restriction de l'accès aux postes de travail en fonction des qualités considérées comme nécessaires à l'activité professionnelle (titre, ancienneté, nationalité, etc.).

Selon cette approche, un marché fermé est un « arrangement institutionnel» alternatif au marché dans la mise en œuvre de transactions. Or, la survie des pratiques institutionnalisées dépend de leur capacité d'adaptation et de leur capacité à rester légitimes. Une des caractéristiques des marchés du travail fermés est donc d'être une construction sociale par nature instable dans la mesure où la stabilité des règles d'emploi et leur agencement dépendent fondamentalement de la stabilité des compromis établis entre les acteurs du marché du travail. Ainsi, tout facteur susceptible de porter atteinte au système d'opportunité des acteurs va faire naître des conflits et rendre nécessaire l'évolution du marché fermé. Dès lors, l'étude de leur dynamique suppose le repérage et la compréhension de ces facteurs d'instabilité. On sait, par exemple, que tous les facteurs affectant la rareté des compétences vont venir modifier le rapport entre l'offre et la demande de travail.

Qui sont les acteurs qui construisent ces «monopoles $d u$ marché du travail », qui en régulent l'accès et en organisent la mobilité ? C. Paradeise (1988) affirme que la construction de ces marchés résulte de l'action et de l'interaction de diverses classes d'acteurs: «Les travailleurs, s'ils parviennent à imposer leurs propres normes de recrutement et de promotion; les employeurs, s'ils ressentent la nécessité de s'assurer les services d'une main-d'œuvre rare et non-substituable dans un état de la formation et de la division du travail ; l'État (ou les intérêts dont il est l'expression), s'il veut garantir la société - au nom d'une définition sociale de "l'intérêt général" - contre les dangers auxquels pourrait l'exposer une relation salariale ou marchande incontrôlée. »

Les marchés du travail des professions réglementées constituent donc une des composantes des marchés fermés. Nous proposons de retenir, dans cet article, la définition proposée par le Parlement européen et le Conseil européen dans le cadre de la directive 2005/
36/CE du 7 septembre 2005, relative à la reconnaissance des qualifications professionnelles. Dans l'article 3 de cette directive, on entend par « profession réglementée »: "une activité ou un ensemble d'activités professionnelles dont l'accès, l'exercice ou une des modalités d'exercice est subordonnée directement ou indirectement, en vertu de dispositions législatives, réglementaires ou administratives, à la possession de qualifications professionnelles déterminées; l'utilisation d'un titre professionnel limitée par des dispositions législatives, réglementaires ou administratives aux détenteurs d'une qualification professionnelle donnée constitue notamment une modalité d'exercice. » Dans les trois professions étudiées, l'État va légaliser un monopole professionnel et légitimer la position de pouvoir des travailleurs par rapport à ceux qui sont exclus de ce marché. La justification de la fermeture de ces marchés du travail se fonde sur une reconnaissance de l'utilité sociale et de la compétence reliée à des besoins bien identifiés. Si l'accès des marchés du travail des professions réglementées est fortement contrôlé (numerus clausus, niveau de qualification, stage professionnel, ...), il en est le plus souvent de même des conditions de transmission des entreprises, de la définition des tâches réservées et de l'organisation de la représentation collective. Cette réglementation professionnelle, au demeurant très variée, joue globalement comme «barrières institutionnalisées à l'entrée» pour ceux qui ne remplissent pas les conditions requises.

Enfin, précisons, pour deux des cas étudiés, qu'au sein des marchés du travail de ces professions réglementées (ou organisées par la loi), les marchés du travail de professions dites «libérales» co-existent peu ou prou avec des marchés de salariés. Certains auteurs notent d'ailleurs toute la difficulté à identifier les professions libérales, tant sont absentes les définitions précises ${ }^{4}$; il faut cependant souligner qu'une des caractéristiques des professions libérales, qui les distingue du salariat, est l'indépendance dans l'exercice de l'activité. C'est le cas bien sûr pour les experts-comptables, mais aussi dans une moindre mesure pour les infirmières. Mais le particularisme

\footnotetext{
${ }^{4}$ Nous renvoyons les lecteurs à l'étude réalisée par le Conseil économique et social de la Région des Pays de La Loire (F. Browne de Kilmaine, 1999).
} 
de ces professions tient surtout à la nature des relations entretenues avec les pouvoirs publics. Ainsi, pour reprendre les deux exemples des marchés professionnels cités, les pouvoirs publics ont organisé les professions médicales et les professions paramédicales pour assurer l'intérêt général de santé publique. De même, la création de la profession d'expertcomptable (et des commissaires aux comptes) avait pour objectif de sécuriser les relations économiques. L'importance de l'affiliation à une association professionnelle (de type «Ordre» par exemple pour les professions libérales ; $c f$. encadré 2) doit être perçue comme le signe d'une volonté étatique de faire en sorte que les travailleurs s'identifient à leur profession et non pas à l'organisation qui les emploie. Leur autonomie se reflète alors dans l'existence d'un code de déontologie de la profession auquel leurs pratiques professionnelles doivent être conformes 5 .

\section{LA DYNAMIQUE DE TROIS PROFESSIONS RÉGLEMENTÉES}

Partant de ces caractérisations théoriques générales qui fondent un marché du travail fermé et réglementé, nous allons rendre compte de la façon dont trois d'entre eux font faire évoluer leurs modes de régulation en s' « ouvrant» à des pratiques nouvelles venant modifier les règles existantes. Plus particulièrement, nous illustrerons les réponses apportées par ces professions dont l'accès était jusqu'alors contraint par des règles rigides et qui ont à faire face à une transformation des comportements de l'offre de travail (moindre attrait des métiers correspondants pour les populations dans lesquelles le recrutement s'opérait traditionnellement, attirance de nouvelles populations ; évolution des comportements de mobilité et de carrière). Nous mentionnerons notamment les principales actions mises en place par l'État et les organisations professionnelles patronales pour faire face aux tensions provoquées par ce changement de comportement (transformation des modalités d'accès

\footnotetext{
${ }^{5}$ Cependant, il ne faudrait pas sous-estimer l'hétérogénéité marquée des situations identitaires selon les professions, et à l'intérieur d'une même profession. Ainsi par exemple, pour les infirmiers, les attentes de l'Ordre sont différentes selon le statut de profession libérale ou le statut de salarié de structures hospitalières.
}

\section{Encadré 2 Les Ordres professionnels}

Les pouvoirs publics ont estimé que certaines professions, dites libérales et réglementées, devaient être assujetties à des règles de comportement plus exigeantes que dans les autres champs professionnels. Ces règles sont réunies sous le nom de "déontologie» (" connaissance des devoirs"). Le législateur a confié l'organisation et le contrôle de ces professions à des Ordres professionnels, dirigés par des membres de la profession, élus par leurs pairs. Une quinzaine de professions réglementées ont été dotées d'un Ordre entre 1945 et 1947: les médecins, les pharmaciens, les sages-femmes, les avocats, les experts-comptables, etc. Le dernier Ordre créé est celui des infirmiers, en avril 2007, alors que cette profession ne compte que $15 \%$ de ses effectifs sous statut de profession libérale. Cet ordre fixe des règles de déontologie, mais aussi déploie des missions d'organisation de la formation initiale et continue, d'élaboration de référentiel de compétences, de contrôle des modalités d'accès à la profession et de régulation démographique.

par la formation, rôle de la certification, accès facilité à certaines populations en réduisant le niveau d'exigences, en modifiant les quotas, en ouvrant à des diplômés d'autres filières...). Nous verrons aussi que pour s'adapter à de nouveaux publics, pour les fidéliser, il convient d'élargir les possibilités de carrières internes et de reconnaissance de l'expérience et également d'organiser des passerelles vers d'autres activités pour répondre à des souhaits de mobilité et de carrière.

\section{Les marins-pêcheurs ou comment palier aux carrières courtes}

Les effectifs de marins du secteur des pêches maritimes diminuent régulièrement depuis vingt ans, à un rythme annuel proche de $4 \%{ }^{6}$. On compte

\footnotetext{
${ }^{6}$ Cette baisse semble toutefois s'être ralentie depuis 2002.
} 
aujourd'hui 20000 marins-pêcheurs en activité en France, dont seulement 16000 sont considérés comme embarquant plus de six mois par an. Malgré la baisse de l'emploi, liée en grande partie à la réduction de l'effort de pêche et de la flotte, de nombreux armateurs déclarent être confrontés à des difficultés de recrutement. Près de $10 \%$ affirment même ne pas réussir à embaucher (Podevin G., 2003) ${ }^{7}$. Dans le même temps, le nombre de personnes qui entrent chaque année dans la profession se maintient en moyenne autour de 1000 nouveaux marins. Dans un contexte de décroissance de l'emploi, ce volume de recrutements devrait largement suffire au remplacement des départs à la retraite (et décès) d'environ sept cents par an. Mais, en réalité, les recrutements viennent pour l'essentiel compenser des sorties en cours de vie active de plus en plus précoces et de plus en plus nombreuses. Dans ce secteur, le taux de turnover est supérieur à $10 \%$. Si le phénomène des carrières courtes à la pêche n'est pas nouveau, il a pris une ampleur sans précédent au cours de la dernière décennie. Ainsi, près des deux tiers de marins ayant débuté leur carrière en 1995 avaient quitté la profession sept ans plus tard, dont bon nombre après seulement quelques mois d'activité (Checcaglini A., Podevin G., 2002).

Les multiples raisons de ces abandons et du raccourcissement des carrières sont bien connues : conditions de travail difficiles et dangereuses, temps des marées très longs générant des rythmes terre/mer difficilement conciliables avec une vie familiale, faible perspective de carrière, improbable installation à son compte,... Les niveaux de rémunération, pourtant supérieurs en moyenne à ceux proposés à terre pour des qualifications comparables, ne suffisent plus à compenser ces conditions difficiles.

Or, le système d'emploi spécifique au marché du travail à la pêche ne peut se maintenir sans changement que si les effectifs de main-d'œuvre qui s'y présentent sont suffisants. Dans le cas contraire, un risque de blocage le menace. C'est ce que le développement des carrières courtes va provoquer, en générant un besoin croissant d'arrivées que le système de

\footnotetext{
${ }^{7}$ De récentes estimations réalisées par les comités locaux des pêches donnent un déficit de plus de 1000 marins, dont 300 pour le seul département du Finistère.
}

formation institutionnalisé ne peut complètement satisfaire. Le premier Contrat d'étude prospective réalisé dans ce secteur, en 2002, concluait notamment que le problème n'était pas tant d'attirer de nouvelles recrues, notamment des jeunes, que de les garder et de les fidéliser. Vouloir augmenter les flux de formés sans modifier les modalités d'entrée et la nature des publics recherchés s'est révélé être une impasse. Les fédérations d'armateurs et de patrons de pêche, les pouvoirs publics, mais aussi la plupart des syndicats de marins ont alors défendu le principe d'une « ouverture » du système d'emploi, jugé trop rigide et trop fermé.

En effet, le secteur des pêches maritimes est un espace régi par des règles spécifiques de recrutement et de mobilité, où le diplôme, la relation entre les brevets de navigation, l'ancienneté et le poste de travail jouent un rôle central. Comme dans tout système fermé, la formation apparaît comme la véritable « charpente » du marché du travail à la pêche, régulant accès aux emplois et promotions. Le système repose sur les brevets imposés dès l'entrée dans la profession. Depuis 1975, aucun marin ne peut embarquer s'il n'est titulaire d'un CAP ou BEP (certificat d'aptitude professionnelle et brevet d'études professionnelles) maritime, ou, plus récemment, d'un Certificat d'initiation nautique $(\mathrm{CIN})^{8}$. Puis, pour faire carrière, le marin-pêcheur devra se former en alternant acquisition de savoirs et savoir-faire en école, et cheminement tout au long d'un cycle d'expériences. L'articulation entre formation initiale et formation continue y est, de ce fait, singulière : la plupart des brevets de commandement relèvent de la formation continue, obligatoire pour qui veut progresser dans la carrière. De plus, les adaptations du système de formation sont lourdes à gérer, puisque toute évolution doit se traduire réglementairement. D'où un décalage fréquent entre le brevet, à évolution lente, et le métier aux compétences à évolution plus rapide.

\footnotetext{
${ }^{8}$ Instauré il y a quinze ans, le CIN se prépare seulement en huit semaines et s'adresse à des jeunes de plus de vingt ans et disposant déjà d'un niveau $\mathrm{V}$ de formation (CAP ou BEP dans les spécialités industrielles). Cette voie d'accès vient concurrencer le CAP et le BEP maritimes. La mise en place récente d'une CIN par alternance, si elle renforce la professionnalisation de ce titre, tend aussi à intensifier cette concurrence.
} 
C'est pourquoi l'État et les organisations professionnelles du secteur ont introduit, depuis longtemps, de la souplesse dans ce système rigide, par des pratiques « dérogataires» visant à autoriser des navires en sous-effectifs à partir en mer; ou bien, plus souvent encore, en acceptant des « faisant fonction » temporaires pour des marins titulaires de brevets inférieurs à ceux normalement requis pour la fonction exercée. Mais ces formes «d'accommodement» ont leurs limites et ne permettent pas que s'instaure durablement un nouveau modèle de régulation interne.

Depuis cinq ans, de nouvelles marges de manœuvre semblent se dégager dans ce secteur, qui continue pourtant de subir des contraintes économiques très fortes. Ces marges de manœuvre s'inscrivent dans une réforme de l'enseignement maritime. Celle-ci a porté tout d'abord sur l'intégration des établissements d'enseignement maritime au sein de l'enseignement public, en les sortant de leur cadre associatif antérieur. Au sein de la Direction des Affaires maritimes a été créé, fin 2000, un Bureau de l'emploi et de la formation qui est devenu la cheville ouvrière de la Commission nationale de l'emploi maritime, et du Comité spécialisé de la formation professionnelle. Ainsi outillée, l'administration a engagé des réformes en synergie avec les représentants de la profession, armateurs et patrons de pêche. Elle a demandé à ces derniers d'assurer leur part des réformes en améliorant les conditions de vie et de travail à bord des navires, mais aussi en agissant sur l'accueil, l'insertion et la fidélisation des jeunes, et plus généralement en s'impliquant davantage dans la formation, notamment par voie d'apprentissage. La mise en place récente d'une CPNE (Commission paritaire nationale pour l'emploi), associant l'ensemble des partenaires sociaux, suite aux recommandations du CEP pêche de 2002, vient compléter le dispositif institutionnel public et paritaire et fournit à la politique d'emploi et de formation les instruments qui lui manquaient.

D'autres réformes illustrent les changements profonds qui affectent le système. C'est ainsi que la filière de formation à la pêche est complétée, depuis 2005, par la mise en place, au-delà du BEP, d'un baccalauréat professionnel venant prolonger le cursus - et auquel sont associées de nouvelles prérogatives de commandement après un temps de navigation. Par ailleurs, pour des candidats adultes ayant déjà un parcours professionnel, voire une qualification non maritime, le renforcement du CIN, bien qu'il garde l'image d'un certificat «faute de mieux », joue un rôle déterminant pour l'insertion de nouveaux publics provenant de viviers non traditionnels. La signature récente (en 2006) d'un accord de branche portant sur l'organisation des contrats de professionnalisation ${ }^{9}$ devrait conférer à ces CIN une dimension professionnelle accrue, notamment par une implication plus grande des employeurs du secteur sous forme de tutorat renforcé. Par ailleurs, la nécessité de démarrer un contrat de professionnalisation par une " marée découverte », c'est-à-dire par un embarquement dans des conditions réelles, devrait lever toute illusion sur le métier et limiter ainsi les abandons en cours de formation. Pour les «faisant fonction ", dérogataires du système d'emploi et de brevets, mais ayant acquis une expérience sur des postes nécessitant une qualification qu'ils ne possédaient pas encore, la VAE (validation des acquis de l'expérience) permet, depuis cinq ans, l'accès à des brevets supérieurs avec dispense totale ou partielle du retour à l'école. Toutefois, cette nouvelle procédure de la VAE ne semble pas encore très probante pour le secteur de la pêche. Enfin, entreprise il y a trois ans, la réforme concomitante des formations et des brevets de la filière « Marine Marchande » et de la filière "Plaisance et Nautisme », par les passerelles qu'elle autorise désormais, facilite les mobilités d'un secteur à l'autre.

Toutefois, pour les acteurs de la profession, cette «ouverture» à l'entrée du marché du travail est apparue inséparable d'une «ouverture » à la sortie. Cette dernière étant alors présentée comme une condition de l'amélioration de l'image du secteur supposée attirer davantage de jeunes. Face à des carrières devenues structurellement plus courtes, il est nécessaire d'anticiper et d'instrumenter les reconversions à terre. Mais permettre à certaines qualifications maritimes de se valoriser à l'extérieur

\footnotetext{
${ }^{9}$ Les contrats de professionnalisation, introduits par la loi de mai 2004 sur la formation professionnelle tout au long de la vie, ont succédé aux anciens contrats d'alternance, notamment aux contrats de qualification. Les contrats de professionnalisation, d'une durée de 6 à 12 mois (exceptionnellement 24 mois), sont assortis d'un minimum de formation en alternance devant déboucher sur une certification
} 
suppose en amont de concevoir des formations qui ne s'enferment pas dans l'acquisition de savoirs trop spécifiques. L'ouverture des contenus de formation, à laquelle les syndicats professionnels ont été associés, vers de nouveaux champs de compétences (gestion, management, droit, biologie de la ressource, ...) devrait contribuer à valoriser la filière de formation à la pêche, en la faisant apparaître, pour les jeunes et leurs familles, comme une filière porteuse d'un avenir professionnel.

\section{Les infirmières : forte croissance de la demande de travail et réorganisation des activités}

Le marché du travail des infirmières ${ }^{10}$ est aussi un marché fortement réglementé et contrôlé par l'État, tant dans ses modalités d'accès (rappelons néanmoins qu'il existe une différence majeure avec le secteur des marins, puisque la profession d'infirmière est une profession non seulement fermée, mais aussi soumise à une régulation quantitative imposée par l'État sous forme de quota annuel d'entrée, dans le cadre de la régulation globale et spatiale des professions de santé) que dans les modes de promotion et de déroulement de carrière. Comme celui des marins-pêcheurs, il rencontre aujourd'hui des limites car il ne trouve plus à s'alimenter et à se renouveler dans des conditions suffisantes. De façon récurrente depuis une dizaine d'années, on y constate en effet des difficultés de recrutement, voire de réelles pénuries $^{11}$. Certaines estimations avancent le chiffre d'un déficit proche de 20000 infirmières pour la France.

À la différence du secteur de la pêche cependant, ces tensions se situent ici dans un contexte de forte croissance des effectifs. Ainsi cette profession, qui comptait 280000 individus en 1982, en totalise aujourd'hui 470 000, soit une progression de $69 \%$ en 25 ans. La majorité des infirmières $(70 \%)$ travaille en structure hospitalière, seules $14 \%$ des infirmières exercent en libéral. Mais cette forte progression paraît insuffisante pour satisfaire des besoins estimés, à l'horizon 2015, à 170000 infirmières

\footnotetext{
${ }^{10}$ Par commodité de langage, nous parlerons des infirmières qui représentent près de $90 \%$ de la profession... d'infirmier.

${ }^{11}$ Bien que l'administration des personnels de santé utilise plutôt le terme de tension que de pénurie, tant les besoins non satisfaits sont difficiles à évaluer.
}

supplémentaires. Si de tels besoins se justifient en premier lieu par la compensation des départs à la retraite qui vont s'accélérer dans les dix ans à venir ${ }^{12}$, ils résultent aussi en partie du développement du temps partiel sur les dernières années, singulièrement élevé dans ce secteur ${ }^{13}$, et de la réduction du temps de travail (RTT) par le passage aux 35 heures. Mise en œuvre entre 2000 et 2002, la RTT aurait, dans ce secteur (établissements privés et publics), absorbé plus des deux tiers de l'accroissement du potentiel de travail, qui ne se serait plus accru alors que de $1 \%$ par an ${ }^{14}$.

En outre, les abandons en cours de formation sont nombreux, avec une proportion significative d'élèves - près de $20 \%$ aujourd'hui - qui n'achèveront pas leur cursus (Marquier, 2006).

Globalement, la forte croissance de la demande de travail conjuguée à la réorganisation des activités expliquent les tensions dans les recrutements et les difficultés à pourvoir les besoins. Et, là aussi, le système de formation professionnelle spécifique à cette profession ne peut se maintenir sans rupture que si le nombre de candidats à l'entrée des instituts de formation reste suffisant.

Dans un premier temps, la réponse de l'administration a été de relever les quotas de recrutements des écoles d'infirmières (Instituts de formation aux soins infirmiers). Ces quotas ont largement évolué ces dernières années, passant de 22000 inscrits en première année, il y a dix ans, à 30000 aujourd'hui. Le flux d'élèves sortant diplômés après 37 mois et demi de formation est quant à lui passé, sur la même période, de 13800 à 20000 . Cet effort quantitatif paraît encore insuffisant, d'autant que certaines écoles rencontrent des difficultés à remplir les places ouvertes. Les tensions sur les recrutements des infirmières commencent donc par les difficultés à recruter... les élèves infirmières $(6 \%$ des places n'ont pas été pourvues en 2003). Mais surtout, l'augmentation des quotas focalise les questions du

\footnotetext{
${ }^{12}$ L'âge moyen des infirmières, qui était de 34 ans en 1982, est aujourd'hui proche de 45 ans. Sur les quatre dernières années, le poids des plus de 50 ans est passé de $24 \%$ à $30 \%$.

${ }^{13}$ Près d'un quart des effectifs serait à temps partiel. $C f$. Acker F. (2003), «Les infirmières: une profession en crise?» in De Kervasdoué J., La crise des professions de santé, Dunod.

${ }^{14}$ Voir rapport ONDPS 2004, tome sur la profession d'infirmière.
} 
recrutement sur le seul volet des volumes d'entrée aux écoles et occulte d'autres phénomènes plus qualitatifs qui seraient en mesure de fidéliser la main-d'œuvre, tels que les conditions de travail et de déroulement de carrière, de mobilité entre activité et statut et de reconnaissance salariale. Par ailleurs, des mesures voulues par l'État, visant le recours aux nonnationaux (infirmières polonaises, espagnoles, ...), ou bien encore la mise en place de dispositifs de « retour à l'emploi » d'infirmières ayant arrêté tôt leur carrière, se sont révélées peu efficaces, puisque elles n'ont concerné que quelques centaines de personnes (rapports ONDPS).

En revanche, l'accès aux cursus de formations pour de nouveaux publics de candidats, plus âgés et possédant une expérience professionnelle, se développe, notamment ces dernières années, avec une part croissante de ces nouveaux entrants en formation (en 2004, cette part était de $32 \%$, cf. Marquier, 2006). Ces nouveaux publics plus âgés, et aussi plus motivés, connaissant déjà pour certains la réalité du métier (notamment ceux qui viennent de la catégorie des aides-soignantes), devraient entraîner une diminution des abandons en cours d'études et réduire les départs précoces. La volonté de favoriser la promotion de certains agents pourrait contribuer également à la régulation des effectifs. Ainsi, le dispositif de VAE, dont devraient pouvoir bénéficier prochainement les aides-soignantes, ouvrirait une nouvelle voie d'accès au diplôme. La VAE permettra aussi d'effectuer le reclassement des "faisant fonction », nombreux dans certaines spécialisations (infirmières de bloc et cadres de santé notamment).

Enfin, la mise en place annoncée d'un schéma universitaire pour la formation initiale des professions paramédicale, inscrivant celle-ci dans le système européen LMD (licence-master-doctorat), devrait ouvrir des perspectives d'évolution professionnelle plus large vers l'expertise ou la recherche dans les sciences de l'infirmière, par exemple, comme cela est déjà le cas dans de nombreux pays, et contribuer ainsi à rendre plus attractives, parce que plus diversifiées, les carrières d'infirmières...

Dans ce contexte, l'État va jouer un rôle de premier plan pour tenter de desserrer les multiples contraintes qui pèsent sur cette profession. Si l'État est lui-même l'employeur majoritaire de cette profession, à travers la fonction publique hospitalière, il va chercher à entraîner avec lui, notamment par la création récente d'un Ordre des infirmiers (encadré 2), l'ensemble des acteurs de la profession; tâche ardue pour une profession au paysage institutionnel complexe et très morcelé - avec plus de 150 associations, syndicats, groupements professionnels - qui semble très divisée sur le rôle et l'utilité de cet Ordre. Pour l'instant, la régulation démographique reste la prérogative d'instances nationales où siègent les partenaires sociaux et l'État. Il s'agit principalement du Conseil supérieur des professions paramédicales - remplacé récemment par le Haut conseil des professions paramédicales - et du Conseil supérieur de la fonction publique hospitalière. Ce Haut Conseil, créé en même temps que l'Ordre des infirmiers, est l'interlocuteur privilégié de l'État et des Ordres des professions médicales, mais aussi de la Haute autorité de santé - autorité publique indépendante à caractère scientifique qui évalue les pratiques professionnelles - et des institutions européennes.

Au total, la perspective, en construction, d'une multiplication des passerelles entre activités et services, entre statuts et spécialités, l'usage de la VAE et l'élargissement du champ professionnel vers des taches jusque-là du domaine réservé des médecins (Robelet M., Serré M., Bourgueil Y., 2005), sont, comme dans le marché du travail à la pêche, autant de formes nouvelles qui doivent redonner de la souplesse à la régulation et à la gestion des effectifs et faciliter l'organisation d'éventuelles reconversions tant internes qu'externes...

\section{Les experts-comptables: un monopole en question}

En France, la profession comptable libérale se singularise à la fois par l'existence de deux organisations professionnelles et par la présence des pouvoirs publics auprès de ces deux organisations. Les deux activités, expertise comptable et commissariat aux comptes, ont en commun les caractéristiques de base des professions réglementées: protection du titre, activité réservée, inscription obligatoire et responsabilité professionnelle. Nous ne traitons ici que du marché du travail des expertscomptables. 
Pour porter le titre d'expert-comptable et exercer leur activité à titre libéral, les experts-comptables doivent obligatoirement être titulaires du diplôme d'expertise comptable (DEC) et être inscrits à l'Ordre des experts-comptables. Créé par la loi en 1945 et placé sous la tutelle du ministère de l'Économie, des Finances et du Budget, l'Ordre représente, défend et organise la profession libérale ${ }^{15}$. C'est un acteur essentiel de la régulation du marché du travail de l'expertise comptable.

L'évolution du mode d'exercice libéral, sous l'effet de l'intensification de la concurrence, de l'ouverture internationale, de l'inter-professionnalisation et d'une démographie défavorable, conduit les différents acteurs à envisager d'importantes évolutions dans le mode d'accès au marché et dans le mode d'exercice de l'activité, tout en préservant les caractéristiques d'un marché fermé du travail.

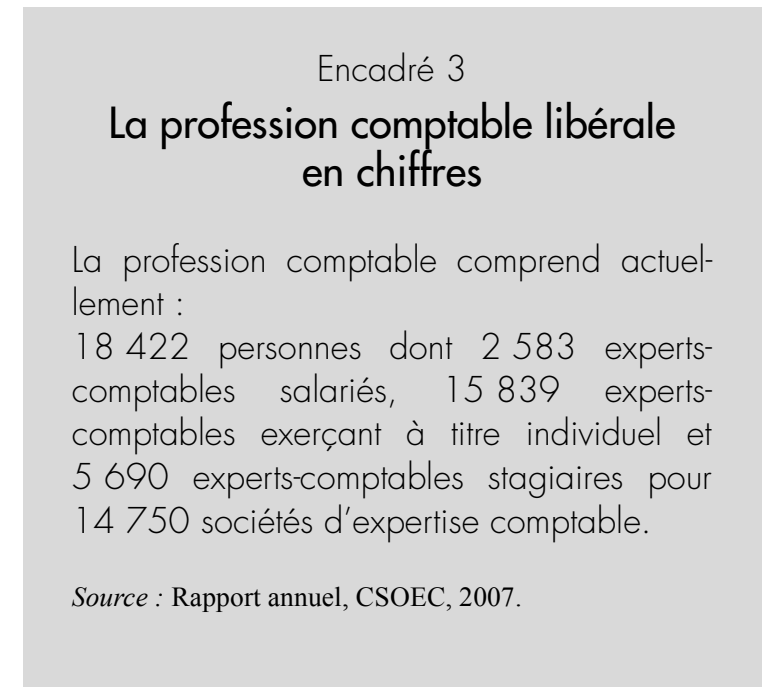

Actuellement, la profession compte $50 \%$ d'inscrits de 50 ans et plus et l'année 2005 a marqué les premiers départs massifs à la retraite. Cette pyramide des âges pose donc la question du renouvellement nécessaire des diplômés inscrits. Mais la désaffection des filières comptables et une baisse du nombre d'inscrits aux épreuves du DEC et des experts-

${ }^{15}$ L'ordre des experts-comptables est représenté par le Conseil supérieur, constitué de 66 membres dont 22 représentent les Conseils régionaux qui ont pour mission de garantir le bon fonctionnement de la profession sur le terrain. comptables stagiaires, depuis 2000, aggravent cette situation (CSOEC, 2006).

À ce problème d'ordre «quantitatif» s'ajoute une exigence de plus en plus forte des cabinets en matière de recrutement, liée à la pression des clients et au développement de nouvelles activités. Si les missions comptables demeurent en effet la base même de l'exercice professionnel, l'activité s'est largement diversifiée, d'une part en développant le conseil, d'autre part en élargissant le champ d'intervention des experts-comptables dans le domaine social et fiscal, dans le monde agricole, le secteur public et le monde associatif. Ce développement d'activités diverses soulève la question de l'interprofessionnalité et de la pluri-activité. L'interprofession peut être définie comme la mise en commun simultanée ou successive de disciplines, à un moment donné ou de façon permanente, au profit d'un client. C'est ce que font déjà de nombreux cabinets internationaux (KPMG, PWC, Deloitte) en France. Confrontés à cette évolution de leur "périmètre » professionnel, de nombreux cabinets nationaux rencontrent parfois de vives difficultés dans le renouvellement des équipes et la recherche de nouveaux profils.

Prendre la mesure des tensions sur le marché du travail des experts-comptables suppose d'étudier sa place et sa dynamique au sein du marché du travail de la profession comptable dans son ensemble, dont il est le segment réglementé. On constate alors qu'à ce besoin de renouvellement des actifs sur le marché $\mathrm{du}$ travail de la profession comptable libérale se conjugue une forte croissance des besoins sur le marché de la profession comptable, quels que soient son mode et son lieu d'exercice (encadré 4). Ainsi, et même si aucune statistique ne permet actuellement d'en mesurer réellement l'effectivité, il semble que les nouvelles générations d'experts-comptables développent de nouveaux modes de rapport au travail et de nouvelles attentes en termes d'exercice de la profession et de carrière professionnelle. Les jeunes diplômés semblent en effet de plus en plus nombreux à être attirés par le salariat. Les études de comptabilité débouchent sur un grand nombre de métiers très cloisonnés. Les titulaires du DEC peuvent opter pour l'expertise comptable, l'entreprise, ou le commissariat aux comptes. Bien que leur diplôme soit identique à celui des professionnels libéraux, les 


\section{Encadré 4 \\ Expertise comptable : conditions de diplôme et mode d'exercice}

La formation de l'expert-comptable s'organise en trois étapes, chacune validée par un diplôme correspondant à différents niveaux de qualification :

- le diplôme de comptabilité et de gestion (DCG), niveau Licence ;

- le diplôme supérieur de comptabilité et de gestion (DSCG), niveau Master ;

- le diplôme d'expertise comptable (DEC), niveau Doctorat.

Le DEC est décerné aux titulaires du DSCG qui, après avoir accompli un stage professionnel de trois ans, ont passé avec succès des épreuves finales.

À l'issue de sa formation, le DEC permet d'exercer en qualité d'expert-comptable à condition de s'inscrire à l'Ordre des experts-comptables. II permet également d'exercer en qualité de "commissaire au compte » sous réserve d'être inscrit auprès d'une compagnie de commissaires au compte. Environ $90 \%$ des experts-comptables sont commissaires au compte. Enfin, le DEC peut donner accès à un poste de cadre supérieur dans une entreprise

La profession libérale d'expert-comptable peut être exercée sous forme d'entreprise individuelle, de société commerciale ou civile ainsi que sous forme d'association de gestion et de comptabilité (depuis l'ordonnance de mars 2004).

diplômés d'expertise comptable en entreprise ne sont pas membres de l'Ordre des experts-comptables. Ils n'appartiennent pas à la même communauté professionnelle et peuvent même être des « rivaux », dans un contexte où les employeurs doivent faire face à des difficultés de recrutement.

L'attractivité d'une carrière en entreprise - cette dernière permettrait d'échapper aux contraintes inhérentes au statut et à l'activité de profession libérale engendre ainsi une « fuite » des diplômés et apparaît comme une explication des tensions sur le marché du travail des experts-comptables libéraux. Une part croissante de jeunes inscrits quitte la profession afin d'exercer comme salariés d'entreprise après avoir acquis de bonnes connaissances et compétences en cabinet d'expertise comptable. L'heure est donc à l'état des lieux et à la réflexion prospective pour que des réponses appropriées puissent être envisagées. Les associations professionnelles ANECS (Association nationale des experts-comptables et commissaires aux comptes stagiaires) et CJEC (Club des jeunes experts-comptables et commissaires aux comptes), en collaboration avec le Conseil supérieur de l'Ordre des experts-comptables et l'Académie des sciences et techniques comptables et financières, travaillent actuellement à une cartographie des diplômés non inscrits à l'Ordre afin d'estimer le nombre de repreneurs potentiels des cabinets et assurer le renouvellement.

Le marché du travail des experts-comptables est donc actuellement soumis à de multiples tensions qui le contraignent à évoluer en assouplissant sa «fermeture» et son étanchéité. Les différents acteurs, pouvoirs publics, Ordres et syndicats, sont conduits à redéfinir les règles relatives au mode d'accès au marché et au mode d'exercice de l'activité, tout en préservant les caractéristiques d'un marché fermé du travail.

S'agissant des conditions d'établissement et d'exercice, l'ordonnance du 25 mars 2004 a aménagé les dispositions de l'ordonnance du 19 septembre 1945 portant institution de l'Ordre des experts-comptables et réglementant le titre et la profession. Les barrières à l'entrée sur le marché du travail des expertscomptables ont été levées, puisque l'exercice de l'activité d'expertise comptable est désormais possible sous forme d'association de gestion et de comptabilité (encadré 4). Concernant les conditions de diplôme, une réforme importante des diplômes 
comptables de l'État a modifié, en décembre 2006, les voies de formation et donc l'accès au marché du travail. Cette réforme des études menant à l'expertise comptable permet d'harmoniser le cursus des diplômes comptables de l'État avec le schéma européen de l'enseignement supérieur LMD. Mais, audelà de cet objectif, elle répond au souhait de la profession comptable libérale d'élargir le recrutement à des candidats ayant des compétences de haut niveau et des profils plus diversifiés. Le nouveau diplôme renforce le niveau de qualification «tout en offrant un attrait plus grand aux lauréats des grandes écoles » (CSOEC, 2005). Si la filière classique de formation consiste à préparer successivement des diplômés nationaux au sein d'établissements d'enseignement supérieur public ou privé, d'autres voies sont possibles et leur développement semble être actuellement perçu par la profession comme une solution au renouvellement et au souhait de recruter des profils diversifiés. Il est ainsi possible d'intégrer le cursus comptable aux différents niveaux et des équivalences sont prévues à partir d'autres diplômes (DUT - diplôme universitaire de technologie -, écoles de commerce, écoles d'ingénieurs, etc.). Enfin, la validation des acquis de l'expérience est une question jugée essentielle et discutée actuellement au sein de la branche par les syndicats professionnels.

Un autre exemple d'évolution du marché du travail a trait à la création de l'Académie des sciences et techniques comptables et financières et de l'Institut des professionnels, associés à l'Ordre des expertscomptables en 2004. En rassemblant autour de l'Ordre les professions françaises de la comptabilité, de la finance, de l'audit, de la gestion, qu'elles œuvrent dans le domaine public ou privé ${ }^{16}$, l'objectif est, pour ces professionnels confrontés à la question de l'interprofessionnalité et de la pluri-activité, de résoudre les tensions entre les différentes activités comptables et leur mode d'exercice. À l'image de la situation des professionnels anglo-saxons, il s'agit de réduire le cloisonnement entre les différents marchés du travail. Ce regroupement a aussi vocation à favoriser le changement d'activité des professionnels

\footnotetext{
${ }^{16}$ L'ensemble des professionnels de la comptabilité, de l'audit, de la finance et de la gestion peuvent accéder au titre de « Professionnel Associé à l'Ordre ».
}

grâce à une fluidification de l'ensemble du fonctionnement $\mathrm{du}$ secteur financier. Proposant une « ouverture à la sortie», il pourrait inciter les jeunes diplômés à rentrer sur le marché du travail de l'expertise comptable sans renoncer à poursuivre un jour une autre carrière.

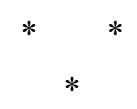

Pour continuer de fonctionner sans à-coups, les marchés professionnels réglementés doivent pouvoir se renouveler régulièrement. Comme le montrent les trois professions étudiées, c'est bien la démographie professionnelle qui va jouer, conjointement au système de formation, un rôle essentiel dans la régulation interne du système. Cette démographie et l'organisation de sa régulation deviennent alors un puissant levier des changements nécessaires qui portent aussi bien sur la composition des recrutements et la gestion des parcours professionnels que sur les identités collectives (Paradeise, 1984).

Si le maintien d'un flux de recrutements suffisant pour garantir une réserve en main-d'œuvre disponible et bien formée reste une priorité, ces recrutements ont tendance, sur la période récente, à changer de nature. Une nouvelle population vient compenser le tarissement des viviers traditionnels de recrutement. Toutefois, du fait de sa socialisation préalable et de son mode d'investissement dans les études, celle-ci se révèle moins disposée à faire de son marché professionnel un cadre unique et spécifique de mobilité. Aussi, les espaces professionnels étudiés sont-ils confrontés autant à l'obligation d'attirer de nouveaux candidats qu'à la nécessité de les fidéliser en leur offrant des opportunités de carrière au-delà des périmètres habituels.

Dans les politiques de fidélisation, la question des carrières semble au centre des solutions possibles pour restaurer une régulation d'ensemble compatible avec les principes de fonctionnement d'un marché fermé. Les carrières se recomposent fortement sous le jeu de multiples contraintes rencontrées pour répondre aux besoins des employeurs, mais ce faisant elles tendent à devenir plus attractives. L'instrumentation de cette fidélisation, présentée ici, renvoie très largement aux politiques patronales institutionnali- 
sées et aux actions de l'État. Le point de vue salarial aurait sans doute fait ressortir, à côté de l'importance des parcours de mobilité, la dimension attractive de la rémunération. Les employeurs ne s'y trompent pas d'ailleurs qui tantôt proposent des compléments de rémunération sous forme de primes ou d'avantages en nature (logement, transport, prise en charge de la formation pour les infirmières) et tantôt garantissent un minimum mensuel de revenu comme à la pêche, où le mode de rémunération traditionnel se fait « à la part », c'est-à-dire de manière très variable en fonction de l'importance des captures et du prix de vente sur les criées.

C'est dans ce sens que travaillent les acteurs des professions étudiées, en cherchant à faciliter les passages entre activités, entre secteurs connexes, ou en introduisant des possibilités de promotions horizontales. Ainsi, parler de l'ouverture des professions ne peut plus se réduire aux seules entrées, mais renvoie aussi aux opportunités en cours de vie professionnelle. Dans ce contexte, reconsidérer la dynamique des marchés ne signifie donc pas ouvrir plus grande les portes des marchés, élargir les voies d'accès, mais signifie beaucoup plus de s'ouvrir à de nouvelles pratiques, à de nouvelles carrières, à de nouvelles possibilités de mobilités tant internes qu'externes. L'élévation des niveaux de formation, l'apparition de nouveaux titres, la création de nouvelles filières, ou bien encore un usage accru des dispositifs de validation des acquis de l'expérience, viennent progressivement offrir de nouvelles perspectives de carrières, moins linéaires et moins cloisonnées.

De façon concomitante, le système de formation connaît des transformations dans le contenu et le déroulement des cursus qui, en évitant une trop grande spécialisation, ouvrent des possibilités de reconversion en dehors du périmètre strict du marché professionnel dont la clôture devient alors relative. Il s'agit de s'ouvrir à de nouvelles possibilités de mobilité à l'intérieur d'un espace qui reste fermé, mais qui s'élargit.
L'apparition progressive de nouvelles règles vient donner de la souplesse aux régulations antérieures, sans toutefois remettre en cause structurellement le système. La crise de légitimité des trois groupes professionnels examinés rend toutefois nécessaire la construction d'une nouvelle «argumentation » des professionnels, à la fois pour maintenir les règles qui organisent la fermeture de leur marché, et pour renouveler les arguments fondateurs et les adapter aux nouvelles pratiques. C'est pourquoi les changements observés peuvent-ils être qualifiés d'organiques plutôt que de structurels (Silvestre J.-J., 1986).

$\mathrm{Au}$ terme de cet article, il est difficile de ne pas évoquer une nouvelle problématique venant impacter la dynamique des marchés professionnels que nous avons étudiée. Au cœur des questions qui émergent au sein des marchés professionnels, nous voyons poindre notamment celle de l'articulation entre « marché interne » d'entreprise ou d'établissement et « marché professionnel », par définition transversal à ces entreprises. En effet, la souplesse retrouvée peut être contrariée localement (en termes d'établissement et d'employeur) par la tendance à une segmentation interne aux espaces professionnels eux-mêmes, induite par des pratiques cherchant la fidélisation de la main-d'œuvre dans un contexte de concurrence accrue entre employeurs. Ce phénomène est amplifié par le développement des savoirs spécifiques et des technicités de plus en plus pointues dans certains des métiers correspondant à ces marchés. Les mobilités entre services, activités, employeurs sont alors rendues plus difficiles (c'est singulièrement le cas pour les infirmières entre services de structures hospitalières). Ce qui nous conduit à nous interroger sur la possibilité de voir apparaître de nouveaux compromis dans un contexte de rivalité de pouvoir entre segments professionnels. Ces évolutions peuvent, à terme, conduire à une déstabilisation des identités collectives produites par les règles initiales des marchés fermés des professions réglementées, et venir déplacer sensiblement les frontières de ces marchés. 
Acker F. (2003), «Les infirmières : une profession en crise ? » in De Kervasdoué J., La crise des professions de santé, Dunod.

Acker F. (2005), «Les reconfigurations du travail infirmier à l'hôpital », Revue Française des Affaires Sociales, $\mathrm{n}^{\circ}$ 1, p. 161-181.

Browne de Kilmaine F. (1999), Les professions libérales, Conseil économique et social des Pays de Loire.

Checcaglini A., Podevin G. (2002), « Des carrières de marins à la pêche de plus en plus courtes qui déstabilisent le marché du travail », in Formation tout au long de la vie et carrières en Europe. ge journée d'études sur l'analyse longitudinale, Documents Céreq, $\mathrm{n}^{\circ} 164$, mai.

Conseil supérieur de l'Ordre des experts-comptables (2006), Rapport annuel 2005.

Déjean F., Saboly M. (2006), « La profession comptable française: entre mimétisme et rivalité ?", Cahiers de recherche, IAE de Toulouse, $\mathrm{n}^{\circ} 175$.

Demazière D. (2008), « L'ancien, l'établi, l'émergent et le nouveau: quelle dynamique des activités professionnelles?», Formation Emploi, $\mathrm{n}^{\circ} 101$, pp. 41-54.

Delahaye-Guillocheau V., Flamant P., Jeandet Mengual E., Audige T. (2004), Gestion des âges dans le secteur sanitaire et social, L'exemple de la filière soignante et des métiers de la personne, Rapport IGAS, n 2004057.

Doeringer P., Piore, M. (1971), Internal labor markets and manpower analysis, Heath Lexington Books, Lexington, Massachussets.

Lamanthe A. (2005), «Les transformations du marché du travail : un éclairage à partir de l'analyse des décalages entre offre et demande dans un système productif localisé », Sociologie du travail, $n^{\circ} 47$.

Le Corre S. (2001), Transformation des marchés du travail et perspectives d'analyse. Réflexions à partir $d u$ cas de la grande distribution alimentaire, Actes des VIIIes journées de sociologie du travail, Aix-enProvence, pp. 139-147.

Marquier R. (2006), Les étudiants en soins infirmiers en 2004, DRESS.

Moysan-Louazel A., Podevin G. (2005), «Quand croissance de l'emploi et vieillissement des actifs conduisent un OPCA à faire évoluer ses services : le cas du secteur sanitaire, social et médico-social », in Joël M-E. et Witter J. (Éds), Économie du vieillissement, âge et emploi, L'Harmattan.

Osty F. (2003), Le désir de métier, engagement, identité et reconnaissance au travail, Presses Universitaires de Rennes.

Paradeise C. (1984), « La marine marchande française, une profession fermée ? », Revue Française de Sociologie.

Paradeise C. (1987), « Des savoirs aux compétences : qualification et régulation des marchés du travail », Sociologie du travail, XXIX, 1, pp. 35-46.

Paradeise C. (1988), «Les professions comme marchés du travail fermé », Sociologie et Sociétés, vol. XX, $\mathrm{n}^{\circ} 2$, octobre.

Pinaud M. (2004), Le recrutement, la formation et la professionnalisation des salariés du secteur sanitaire et social, Avis et Rapports du Conseil économique et social, République française.

Podevin G. (coord.) (2003), Le secteur des pêches maritimes, «Prospective Formation Emploi», La Documentation française.

ONDPS (2006), Rapport annuel de l'Observatoire national de la démographie des professions de santé- 
2005. Synthèse générale - Tome 1 : Les effectifs, l'activité et la répartition des professionnels de santé - Tome 2: Analyse de deux professions, pharmaciens, infirmiers - La Documentation française.

Robelet M., Serré M., Bourgueil Y. (2005), «La coordination dans les réseaux de santé : entre logiques gestionnaires et dynamiques professionnelles ", Revue Française des Affaires Sociales, $\mathrm{n}^{\circ}$ 1, pp. 233260.

Silvestre J.-J. (1986), « Marchés du travail et crise économique : de la mobilité à la flexibilité », Formation Emploi, ${ }^{\circ} 14$, pp. 54-61.

\title{
Résumé
}

\section{Une nouvelle dynamique des marchés du travail réglementés, à l'aune de trois professions}

\author{
Anne Moysan-Louazel et Gérard Podevin
}

Cet article propose d'étudier la dynamique de certains marchés du travail à caractère réglementé. De nombreux indices laissent penser que plusieurs de ces marchés, confrontés autant à l'obligation d'attirer de nouveaux candidats qu'à la nécessité de les fidéliser, subissent, sur la période récente, de profondes transformations. Celles-ci sont particulièrement visibles pour des professions encadrées par la loi auxquelles l'article s'intéresse plus particulièrement. Pour autant, comme nous tentons de le montrer, les changements qui affectent certaines règles ne remettent pas en cause le caractère fermé de ces marchés. Parler de l'ouverture de ces professions ne peut se limiter aux seules entrées mais signifie aussi de s'ouvrir davantage à de nouvelles pratiques, à de nouvelles formes de carrières et à de nouvelles mobilités dans les champs professionnels correspondants.

\section{Mot clés}

Marché du travail, marin-pêcheur, infirmier, expert-comptable, organisation professionnelle Journal of Economic Literature : J 40 